\title{
Anatomical variations detected during ultrasound-guided interscalene brachial plexus block and clinical implications
}

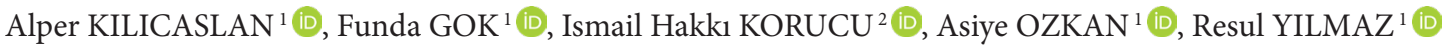 \\ ${ }^{1}$ Department of Anaesthesiology and Reanimation, Meram Medical Faculty, Necmettin Erbakan University, Konya, Turkey. \\ ${ }^{2}$ Department of Orthopedics and Traumatology, Meram Medical Faculty, Necmettin Erbakan University, Konya, Turkey. \\ Corresponding Author: Alper KILICASLAN \\ E-mail: dralperkilicaslan@gmail.com
}

Submitted: $10.06 .2020 \quad$ Accepted: 27.08.2020

\section{ABSTRACT}

Objective: Our aim was to evaluate the anatomic variations detected during ultrasound-guided interscalene brachial plexus block (US-ISB) and present their clinical implications.

Materials and Methods: After the ethical approval for the study was obtained from the local ethics committee, the files and US records of patients who underwent US-ISB for anesthesia of the shoulder surgery were retrospectively analyzed.

Results: Anatomical variations which were considered to affect the block technique were detected in 13 (11.8\%) of 110 patients. C5 cervical root pierced the anterior scalene muscle (ASM) in 4.5\%, and ventral rami of C5 and/or C6 were located in ASM in 3.6\% of patients. There was a muscle bridge between $\mathrm{C} 5$ to $\mathrm{C} 6$ and $\mathrm{C} 5$ to $\mathrm{C} 7$ roots in $1.8 \%$ of the patients. The brachial plexus was located medial to ASM and missing from interscalene groove in $1.8 \%$ of patients. In one case (C5 root was located in ASM), US-ISB resulted in incomplete brachial plexus anesthesia, and so general anesthesia (GA) was performed.

Conclusion: Some of the brachial plexus variations in the interscalene area may be associated with further needle manipulation/ redirection and block failure. We consider that prospective studies including more populations are needed to elucidate the effects of these variations on block parameters.

Keywords: Brachial plexus, Interscalene block, Ultrasonography, Variations

\section{INTRODUCTION}

The ultrasound-guided interscalene brachial plexus block (USISB) is one of the most common procedures for anesthesia and analgesia in the surgery of upper extremities. For the safe and effective regional anesthesia (RA) procedures, it is essential that all relevant anatomical structures must be properly defined. However, the brachial plexus has a complex anatomical structure, and the variations of the brachial plexus associated with its adjacent scalene muscles are common (11-49\%) [1-5]. The variations usually occur as the course of the C5 and/or C6 cervical roots through or anterior to the anterior scalene muscle (ASM) $[1,6]$. Any of such variations can make it difficult to define the anatomical structures and cause confusion in practitioners with insufficient experience and familiar with the normal anatomy.
The variations associated with some nerves, muscles and vascular structures in the interscalene area may affect the technique of the procedure [7-9]. In the presence of such variations, especially in the cephalad approach and ISB conducted with low volume, the scalene muscles can prevent the spread of local anesthesia (LA) by acting as a barrier $[10,11]$. On the other hand, if high-volume LA is performed during ISB, unwanted phrenic nerve blockage and spreading to neighboring anatomical areas, such as epidural space, may occur.

The brachial plexus, needle, spreading of LA and surrounding anatomical structures can be visualized by US [12]. The variations in the interscalene area have been described in many studies; however, the number of the studies investigating the 
clinical effects of these variations is limited, and the majority are case reports.

In the present study, it was aimed to retrospectively evaluate the anatomic variations detected during US-ISB and present their clinical implications.

\section{MATERIALS and METHODS}

After the approval was obtained from the local ethics committee (2019/212) for the present descriptive retrospective study, the files and US records of the patients who underwent US-ISB for anesthesia of the shoulder surgery in a university hospital were analyzed between January 2019 and September 2019. The demographic data of the patients, such as age, gender and ASA the American Society of Anaesthesiologists (ASA) scores in the hospital archive were reviewed.

\section{Interscalene Brachial Plexus Block}

No US-ISB was performed in the patients with infections in the area of surgical intervention, psychiatric disease, neurological damage in the brachial plexus and respiratory failure, and those allergic to local anesthetics.

In our routine clinical practice, US-ISB is performed as described below. The block procedure is carried out by an anesthesiologist experienced in US-guided procedures or senior residents under the supervision of the same anesthesiologist.

While the patients are in the semilateral decubitus position, the $18 \mathrm{MHz}$ linear probe (Esaote MyLab 30 Geneva, Italy) is placed in the supraclavicular fossa. After the brachial plexus is identified as a bright echogenic structure posterolateral to the subclavian artery (bunch of grapes sign), the probe is moved in the cephalic direction up to the interscalene groove by keeping the nerves in the center of the screen. The nerve structures are displayed as the string of hypoechoic circles (traffic light sign) between ASM and middle scalene muscles (MSM) (Figure 1). The sternocleidomastoid muscle, the common carotid artery and the internal jugular vein can be visualized as other main anatomical elements. Finally, the cervical roots are traced towards the cephalad until disappearing into the vertebra.



Figure 1. A) Cross-sectional sonographic image of normal anatomy of the interscalene region, (B) illustrating nerve roots (yellow circles) in between the anterior scalene muscle (ASM) and middle scalene muscle (MSM). SCM: Sternocleidomastoid muscle 
The needle tip is inserted using the in-plane technique and advanced through MSM under direct visualization into the interscalene space, and the nerves are confirmed by stimulating with the nerve stimulator. The spread of LA $(20 \mathrm{~mL}, 0.25 \%$ bupivacaine) around the nerves is confirmed by US. The sensory and motor assessments are performed at 30th minute after the procedure. In our practice, the block was considered unsuccessful if any additional local analgesic or general anesthesia (GA) was used.
Although, the anatomic variations are often visualized during US scanning in the interscalene area, we can mostly identify a needle entry point where we can block the brachial plexus. In cases where the cervival roots are not visualized clearly in the interscalene groove, we perform the low interscalene block, supraclavicular block or GA. The US images of these variations of the brachial plexus are stored in the computer memory of US device for later analyses (Figure 2).
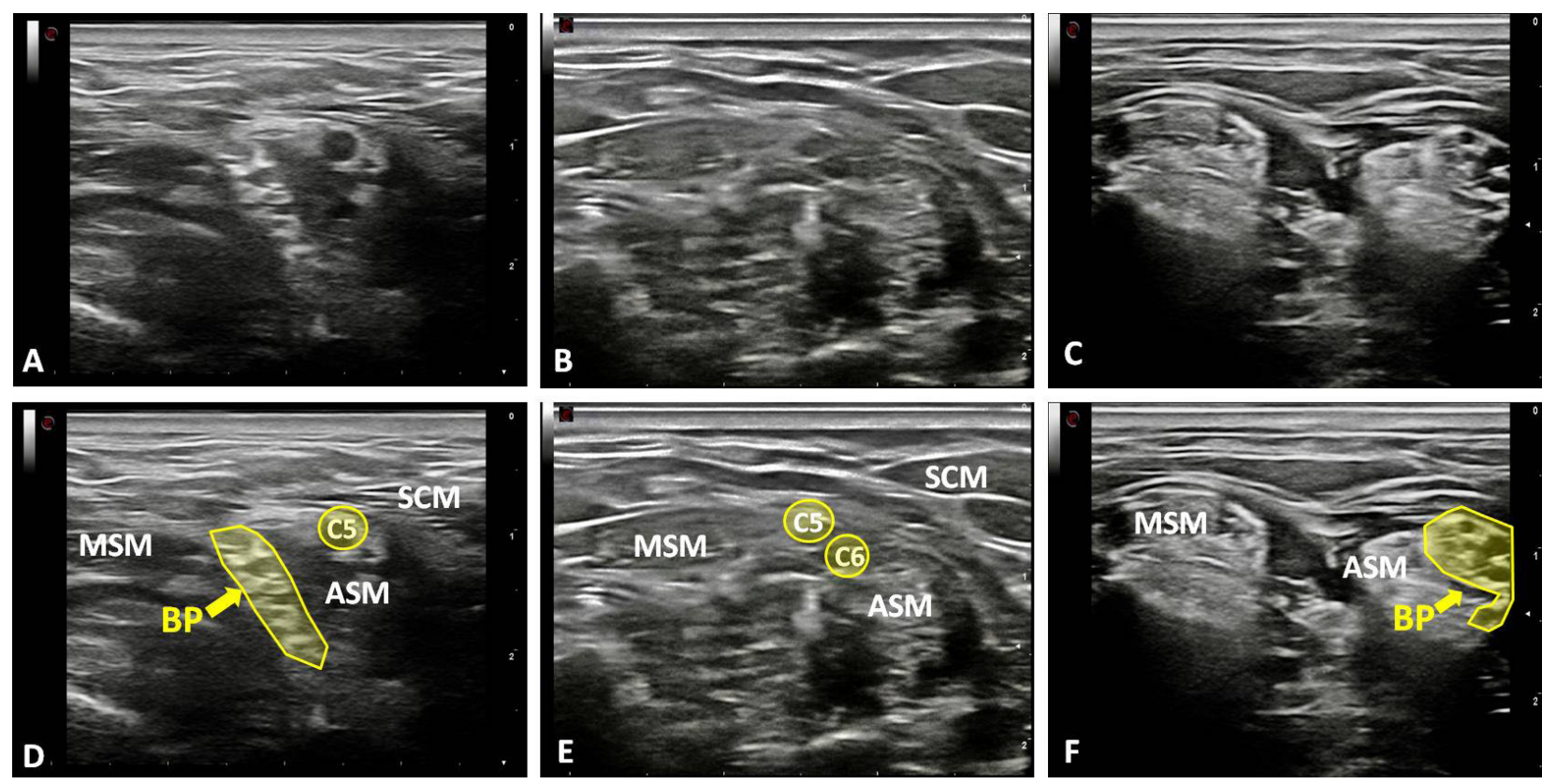

Figure 2. Cross-sectional sonographic images of variations of the interscalene region in $(A, D)$ The ventral ramus of the $C 5$ nerve root is located outside the interscalene groove, pierced the anterior scalene muscle (ASM), the brachial plexus (BP) is encircled by the yellow line. (B,E) The ventral rami of the $C 5$ and C6 roots are located in ASM and a muscle bridge between scalene muscles. The C7 root is not visible in this plane. (C,F) $B P$ (encircled by the yellow line) is located medial to ASM and missing from the interscalene groove.

MSM: Middle scalene muscle, SCM: Sternocleidomastoid muscle

The recordings of these procedures and the data regarding USISB are re-evaluated by two experienced anesthesiologists. The patients with the shortage of data and administered with LA at different doses were excluded out of the study.

\section{Statistical Analysis}

The statistical analysis was performed using the Statistical Package of Social Sciences software for Windows, version 16.0 (SPSS Inc, Chicago, IL, USA). The descriptive statistics of the variables in the study were calculated, and while the continuous quantitative data were given as number, mean and standard deviation (SD) the qualitative data were expressed as number and percentage values. A $\mathrm{p}$ value $<0.05$ was considered to be significant.

\section{RESULTS}

A total of 119 RA records of the patients who underwent US-ISB were retrospectively reviewed, and nine patients were ruled out due to the shortage of data. The demographic and clinical data of the patients are presented in Table I.

The variations, considered to affect the block tecnique, were detected in 13 (11.8\%) out of 110 patients who underwent USISB (Table II). While the ventral rami of C5 cervical spinal nerve pierced the ASM in $4.5 \%$ of the patients (Figure 1A), the ventral rami of C5 and/or C6 were located in ASM in 3.6\% of the cases (Figure 1B). There was a muscle bridge between $\mathrm{C} 5$ to $\mathrm{C} 6$ and $\mathrm{C} 5$ to $\mathrm{C} 7$ roots in $1.8 \%$ of the patients. The brachial plexus was located at medial to ASM, and just lateral to the internal jugular vein (missing from interscalene groove) in $1.8 \%$ of the patients 
(Figure 1C). In addition, the artery crossing or proximal to the roots was detected in $4.5 \%$ of the patients .

Table I. Patients and clinical characteristics

\begin{tabular}{ll}
\hline Value & mean $\pm \mathrm{SD}(\mathrm{n}=110)$ \\
\hline Age (years) & $61.7 \pm 6.5$ \\
\hline Gender $(\mathrm{M} / \mathrm{F})(\%)$ & $(\%) 46(42) / 64(58)$ \\
\hline ASA I/II/III & $19 / 64 / 27$ \\
\hline Duration of surgery (min) & $71.24 \pm 10.2$ \\
\hline Type of surgery & \\
\hline Rotator cuff repair & $79(71.9)$ \\
\hline Bankart repair & $16(14.4)$ \\
\hline Subacromial decompression & $10(9.1)$ \\
\hline Capsular release & $5(4.6)$
\end{tabular}

Data are given as number (proportion) or mean \pm standard deviation. ASA: The American Society of Anesthesiologists, SD: Standard deviation

Table II. Anatomical variations detected during ultrasound-guided interscalene brachial plexus block

\begin{tabular}{lc}
\hline Variations & Total, $\mathbf{n}=\mathbf{1 1 0}(\%)$ \\
\hline C5 pierce ASM & $5(4.5)$ \\
C5 and C6 pierce ASM together & $4(3.6)$ \\
Muscle bridge between roots & $2(1.8)$ \\
Ventral rami (C5-C7) located medial to ASM & $2(1.8)$ \\
An artery crossing roots & $5(4.5)$ \\
Data are given as number (proportion). ASM: Anterior scalene muscle
\end{tabular}

GA was performed in two cases where the brachial plexus was found to be located in the medial of ASM without any needle insertion. In one case, US-ISB resulted in incomplete brachial plexus anesthesia, and so GA was performed. In this case, C5 cervical root was localized in ASM, and the other cervical roots were not clearly visualized. The adequate paresthesia with motor blockade was achieved in all of 97 patients with normal anatomy. No intraoperative or postoperative complications, such as nerve damage, systemic LA toxicity or vascular puncture, were seen in any of the patients.

\section{DISCUSSION}

The main purpose of the present study was to evaluate the anatomical variations of the brachial plexus and adjacent structures in the interscalene area and to investigate their clinical significance. One of the most common variations we identified in the study is the dislocation of C5 ventral ramus out of the interscalene gap. The other is the muscular variations such as a muscle bridge between AMS and MSM separating the cervical roots from each other. In addition, the nerve structures of the entire brachial plexus were seen in the medial part of ASM in two of our patients.

In various studies, the incidence of the variations associated with the brachial plexus and ASM in the interscalene area was reported at different rates ranging between 11-49\% [1-5]. In a study conducted by Harry et al. on cadavers, it was reported that the most common variation was that $\mathrm{C} 5$ and/or C6 ventral rami passed through or over ASM [1]. We observed C5 piercing anomalies in $4.5 \%$ of our patients; even so, the rates of this variation were reported in the studies as $3.1 \%$ by Leonhard et al. [13] , $8 \%$ by Sakamoto et al. [14] and 13\% by Harry et al [1] . However, the current study found a lower frequency of superior trunk piercing anomalies as $3.6 \%$. As opposed to the percentage of $3.6 \%$ in our study, higher frequency of superior trunk piercing anomalies were stated as $15 \%$ and $39 \%$ in the studies by Harry et al. [1] and Leonhard et al. [13], respectively.

In the study performed by Kapral et al. the variations were detected in the interscalene region in $9(11.2 \%)$ out of 80 cases [5]. In another study by Kessler et al., the variation of the brachial plexus was detected in $6(13 \%)$ out of 46 patients (C5 ventral rami passed over the ASM in three patients and passed through ASM in three patients) [6].

In a case report by Chin et al., a patient with a $\mathrm{C} 5$ root located medial to the anterior scalene muscle (ASM) and a C6 root located in the inferomedial was reported [3]. In addition, in another case report performed by Yadav et al., most of the roots originating from the ventral rami were emphasized to be stacked medial to the ASM [15]. This variation, rarely reported so far, was encountered in two (1.8\%) patients in our study. As different from previous cases, our patients would undergo shoulder surgery. Therefore, we performed GA for both cases.

In the study by Gutton et al., at least one variation was detected in the interscalene area through US in 71 (49\%) out of 146 patients [4]. Of these 71 patients, while $8 \%$ had a C5 root anterior to ASM, 33\% had an intramuscularly localized cervical root. Gutton et al. also reported that these variations had no relevant influence on the performance of ISB.

According to our results, however, US-ISB procedure failed in one of the patients determined with a variation. In some of the cases where the variations were visualized, additional needle insertion and more needle manipulation were required to ensure the adequate spread of LA. Anatomical variations may prolong the time of performing blocks. In addition, some variations also resulted in alterations in the anesthesia method.

The clinical effects of such variations become more evident when an anatomical landmark-guided technique is used for ISB. These variations may lead to the limitation of the distribution of injected LA and inadequate analgesia. The combination of anatomical variations may make the brachial plexus block difficult $[7,8]$. Therefore, the use of US becomes important and has a high success rate in the brachial plexus blocks developing with anatomical variations.

Since anatomical variations develop less in the supraclavicular region, the supraclavicular brachial plexus block may be chosen in the patients with variations, if the surgical method is appropriate.

The incomplete brachial plexus block may have occurred due to a number of reasons. We consider that the reason of the failed block is due to restriction of the spread of LA by ASM in this study. 
The anatomical variations may become more important, especially if ISB is performed in a more cephalad position and low volumes of LA solution. In our clinical practice, when these anomalies are observed, we perform ISB $2-3 \mathrm{~cm}$ caudal to the variation point, if possible.

Vascular structures, which will restrict the spread of LA or inhibit the needle advancement such as dorsal scapular artery, can be present between the cervical roots in this region $[8,9]$. While an artery crossing the roots or trunks was revealed in $23 \%$ of the patients in the study by Gutton et al., the rate was observed as $4.5 \%$ in the current study. Even if US is utilized, the practitioners should be more meticulous for intra-arterial injections [16]. The use of Doppler US will be beneficial to identify vessels.

The main limitations of our study are the retrospective nature and that all detailed information could not be available. In addition, the brachial plexus was unilaterally evaluated on the surgical side and we do not have data about the opposite side.

Although, the anatomic variations of the brachial plexus are frequently observed in the interscalene area by US, somehow the block procedure is mostly performed.

Here, the important issue is to differentiate the abnormal sonoanatomy. Some of the brachial plexus variations in the interscalene area may be associated with further needle manipulation/redirection and block failure. It may even be necessary using a different anesthesia technique, depending on the type of the variation. We consider that prospective studies including more populations are needed to elucidate the effects of these variations on block parameters.

\section{Compliance with Ethical Standards}

Ethical approval: The ethical approval was obtained from the local ethics committee (approval number: 2019/212)

Funding: The study was supported by no funds.

Conflict of interest: The authors have no conflicts of interest to declare.

Authors' Contibutions: AK designed the study, gathered and interpreted the data of the patients, performed the statistical analysis and drafted the manuscript. FG interpreted the data and revised the manuscript. IHK conceived the study and revised the manuscript. AO and RY gathered the data of the patients. All authors read and approved the final manuscript.

\section{REFERENCES}

[1] Harry WG, Bennett JD, Guha SC. Scalene muscles and the brachial plexus: anatomical variations and their clinical significance. Clin Anat 1997;10:250-2. doi:10.1002/ (SICI)1098-2353(1997)10:4

[2] Cash CJ, Sardesai AM, Berman LH, et al. Spatial mapping of the brachial plexus using three-dimensional ultrasound. Br J Radiol 2005;78(936):1086-94. doi:10.1259/bjr/36348588

[3] Chin KJ, Niazi A, Chan V. Anomalous brachial plexus anatomy in the supraclavicular region detected by ultrasound. Anesth Analg 2008;107:729-31. doi: 10.1213/ane.0b013e31817dc887
[4] Gutton C, Choquet O, Antonini F, Grossi P. Ultrasoundguided interscalene block: influence of anatomic variations in clinical practice (French). Ann Fr Anesth Reanim 2010; 29 : 770-5. doi: 10.1016/j.annfar.2010.07.013

[5] Kapral S, Greher M, Huber G, Willschke H, Kettner S, Kdolsky $\mathrm{R}$, et al. Ultrasonographic guidance improves the success rate of interscalene brachial plexus blockade. Reg Anesth Pain Med 2008;33:253-8. doi: 10.1016/j.rapm.2007.10.011

[6] Kessler J, Gray AT. Sonography of scalene muscle anomalies for brachial plexus block. Reg Anesth Pain Med 2007;32:1723. doi: 10.1016/j.rapm.2006.09.011

[7] Feigl GC, Pixner T. Combination of variations of the interscalene gap as a pitfall for ultrasound-guided brachial plexus block. Reg Anesth Pain Med 2011;36:523-4. doi: 10.1097/AAP.0b013e31822897f1

[8] Abrahams MS, Panzer O, Atchabahian A, et al. Case Report: Limitation of Local Anesthetic Spread During UltrasoundGuided Interscalene Block. Description of an Anatomic Variant With Clinical Correlation. Reg Anesth Pain Med 2008;33:357-9. doi:10.1016/j.rapm.2008.01.015

[9] Feigl GC, Litz RJ, Marhofer P. Anatomy of the brachial plexus and its implications for daily clinical practice: regional anesthesia is applied anatomy. Reg Anesth Pain Med.2020;45:620-7. doi: 10.1136/rapm-2020-101435

[10] Feigl GC, Pixner T. The cleidoatlanticus muscle: a potential pitfall for the practice of ultrasound guided interscalene brachial plexus block. Surg Radiol Anat 2011; 33: 823-5. doi: 10.1007/s00276.011.0820-Z

[11] Kilicaslan A, Gürkan Y, Tekin M. A brachial plexus variation identified during ultrasound-guided interscalene block. Agri 2012; 24: 194. doi: 10.5505/agri.2012.34966

[12] Koscielniak-Nielsen ZJ. Ultrasound-guided peripheral nerve blocks: what are the benefits? Acta Anaesthesiol Scand 2008;52:727-37. doi: 10.1111/j.1399-6576.2008.01666.x

[13] Leonhard V, Smith, R, Caldwell, G, Smith, HF . Anatomical variations in the brachial plexus roots: implications for diagnosis of neurogenic thoracic outlet syndrome. Annals of Anatomy 2016;206: 21-6. doi.org/10.1016/j.aanat.2016.03.011

[14] Sakamoto Y. Spatial relationships between the morphologies and inner-vations of the scalene and anterior vertebral muscles. Ann Anat 2012 ;194:381-8. doi.org/10.1016/j. aanat.2011.11.004

[15] Yadav N, Saini N, Ayub A.Anatomical variations of interscalene brachial plexus block: Do they really matter? Saudi J Anaesth 2014;8:142-3. doi: 10.4103/1658-354X.125981

[16] VadeBoncouer TR, Weinberg GL, Oswald S, Angelov F. Early detection of intravascular injection during ultrasound-guided supraclavicular brachial plexus block. Reg Anesth Pain Med 2008;33:278-9. doi: 10.1016/j.rapm.2007.12.012 